\title{
Statistical behavior of foreshock Langmuir waves observed by the Cluster wideband data plasma wave receiver
}

\author{
K. Sigsbee ${ }^{1}$, C. A. Kletzing ${ }^{1}$, D. A. Gurnett ${ }^{1}$, J. S. Pickett ${ }^{1}$, A. Balogh ${ }^{2}$, and E. Lucek ${ }^{2}$ \\ ${ }^{1}$ Department of Physics and Astronomy, 203 Van Allen Hall, University of Iowa, Iowa City, IA 52242, USA \\ ${ }^{2}$ Space and Atmospheric Physics Group, The Blackett Laboratory, Imperial College, Prince Consort Road, London, SW7 \\ 2BW, UK
}

Received: 1 October 2003 - Revised: 8 June 2004 - Accepted: 11 June 2004 - Published: 14 July 2004

Part of Special Issue "Spatio-temporal analysis and multipoint measurements in space"

\begin{abstract}
We present the statistics of Langmuir wave amplitudes in the Earth's foreshock using Cluster Wideband Data (WBD) Plasma Wave Receiver electric field waveforms from spacecraft 2, 3 and 4 on 26 March 2002. The largest amplitude Langmuir waves were observed by Cluster near the boundary between the foreshock and solar wind, in agreement with earlier studies. The characteristics of the waves were similar for all three spacecraft, suggesting that variations in foreshock structure must occur on scales greater than the $50-100 \mathrm{~km}$ spacecraft separations. The electric field amplitude probability distributions constructed using waveforms from the Cluster WBD Plasma Wave Receiver generally followed the log-normal statistics predicted by stochastic growth theory for the event studied. Comparison with WBD receiver data from 17 February 2002, when spacecraft 4 was set in a special manual gain mode, suggests nonoptimal auto-ranging of the instrument may have had some influence on the statistics.
\end{abstract}

Key words. Magnetospheric physics (plasma waves and instabilities; solar wind-magnetosphere interactions) - Interplanetary physics (planetary bow shocks)

\section{Introduction}

The foreshock is a region upstream of the Earth's bow shock that is magnetically connected to the bow shock. Within this region, electrons that are reflected and accelerated at the bow shock are convected downstream by the $\boldsymbol{v} \times \boldsymbol{B}$ electric field in the solar wind. The processes occurring near the shock produce electron beams and result in a bump-on-tail electron distribution function (Fitzenreiter et al., 1984). The Langmuir waves observed in the Earth's foreshock are thought to be generated by instabilities due to the bump-on-tail character of the electron beams in this region. The study performed by Filbert and Kellogg (1979) using IMP 6 data showed that

Correspondence to: K. Sigsbee

(kms@beta.physics.uiowa.edu) the wave amplitudes were largest on magnetic field lines that were tangent to the bow shock. Using data from ISEE 1, Etcheto and Faucheux (1984) found that the maximum amplitudes of the Langmuir waves were a few millivolts per meter at the edge of the foreshock and only a few tens to a few hundreds of microvolts per meter inside the foreshock. During a time period when the solar wind magnetic field was unusually constant, Cairns et al. (1997) used ISEE 1 plasma wave data to show that there is a slight offset of the high wave amplitude region from the boundary of the foreshock. Cairns et al. also showed that the high field region is relatively narrow and that the field amplitudes fall off rather slowly at large distances from the foreshock. Other studies using ISEE 1 and Wind data (e.g. Bale et al., 1997; Cairns and Robinson, 1997) have examined the statistical properties of the Langmuir wave amplitudes in order to identify various processes that may operate in the foreshock, such as Langmuir wave collapse (Zakharov, 1972), electrostatic decay (Robinson and Cairns, 1995; Bale et al., 2000), and stochastic growth (Robinson, 1995).

The multiple spacecraft nature of the Cluster mission allows us to investigate the behavior of Langmuir waves in the foreshock in ways that were not possible with earlier data sets. With Cluster, it is possible to compare near instantaneous Langmuir wave amplitudes at different positions within the foreshock and examine motions of the foreshock boundary. Another important feature of the Cluster data set from the Earth's foreshock is that the WBD Plasma Wave Receiver provides electric field waveforms in this region. Earlier studies of Langmuir waves in the Earth's foreshock conducted using data from ISEE (e.g. Greenstadt et al., 1995; Cairns et al., 1997), Geotail (Kasaba et al., 2000), and other spacecraft (e.g. Onsager et al., 1989) relied upon spectral density measurements, which can underestimate the wave amplitudes due to temporal and spectral averaging (see Robinson et al., 1993). The only known exceptions to this are the foreshock studies conducted using waveforms from the Wind spacecraft (e.g. Bale et al., 1997, 2000). In this paper, we present a study of Langmuir waves in the foreshock using 
electric field waveforms from the Cluster WBD Plasma Wave Receivers on spacecraft 2, 3 and 4 on 26 March 2002. This day was selected because there were relatively steady solar wind conditions during most of the time period that Cluster was located in the foreshock. The data from 26 March 2002 will be compared to results from the WBD receivers on Cluster spacecraft 3 and 4 for 17 February 2002 presented in Sigsbee et al. (2004).

\section{Instrumentation}

During the passes through the foreshock reported here, the WBD Plasma Wave Receiver was set in $77 \mathrm{kHz}$ mode. For this bandwidth setting, the instrument obtains two back-toback electric field waveform captures of approximately $5 \mathrm{~ms}$ each every $69.5 \mathrm{~ms}$. The gain of the receiver can be set to amplifications between 0 and $75 \mathrm{~dB}$ in $5 \mathrm{~dB}$ increments. The peak amplitude range is $2.6 \times 10^{-5} \mathrm{mV} / \mathrm{m}$ to $36.9 \mathrm{mV} / \mathrm{m}$ or $123 \mathrm{~dB}$, but the dynamic range in each gain state is $48 \mathrm{~dB}$, corresponding to 8-bit digitization of the waveforms. The gain control can be set in either a fixed-gain mode or an autoranging mode. In fixed-gain mode, the gain can be set to any of the 16 levels between 0 and $75 \mathrm{~dB}$. In the auto-ranging mode, the WBD receiver gain state is adjusted to keep the measured average amplitude within the range of the digitizer at the rate determined by gain update clock. For the full details of the instrument and its operating modes, see Gurnett et al. (1997).

Because the amplitudes of Langmuir waves in the Earth's foreshock can change on time scales of $1 \mathrm{~ms}$ or less, the $0.1 \mathrm{~s}$ time period of the Digital Wave Processor (DWP) (Woolliscroft et al., 1997) gain update clock results in occasional clipping of the waveform peaks when the wave amplitudes exceed the maximum range for a particular gain state. On 17 February 2002, the WBD receiver on spacecraft 4 was operated with the gain manually set to $0 \mathrm{~dB}$. This allowed us to consistently observe waveforms with amplitudes at the maximum range of the instrument $(36.9 \mathrm{mV} / \mathrm{m})$ without clipping of the peaks due to non-optimal auto-ranging and to investigate the effects of the automatic gain control on the waveform statistics. Setting the gain manually to $0 \mathrm{~dB}$ means that we can observe the large amplitude waves without clipping, but will miss low amplitude waves which fall below the minimum amplitude threshold for this gain state at $1.0 \mathrm{mV} / \mathrm{m}$. The calibrated amplitudes increase linearly with the raw values, so waveforms with peak amplitudes less than $10.5 \mathrm{~dB}$ above the zero-level are not well-defined and resemble square waves. Waveforms with amplitudes in the lowest $10.5 \mathrm{~dB}$ of the $48 \mathrm{~dB}$ range for each gain state were ignored.

For Langmuir waves, the wave vector $\boldsymbol{k}$ is parallel to the background magnetic field. Because the WBD receiver antenna is located in the spin plane of the Cluster spacecraft, measurements near the plasma frequency in the foreshock can exhibit an amplitude modulation with a period of half the spacecraft spin period (4s) due to the changing antenna orientation with respect to the background magnetic field. Some studies of Langmuir waves in the foreshock have shown that while the most intense electric fields have wave vectors close to the magnetic field direction, the angular distribution of the largest amplitude waves can be very broad (Bale et al., 2000). However, during the time periods we have studied using Cluster data, the modulation of the wave amplitudes was distinct and well-correlated with the orientation of the antenna with respect to the magnetic field. In our study of Langmuir waves in the Earth's foreshock, we multiplied the waveform amplitudes by the correction factor of $1 / \cos \theta$, where $\theta$ is the angle between the antenna and the magnetic field. Data taken from $78^{\circ} \leq \theta \leq 101^{\circ}$ were rejected because the amplitude correction factor becomes very large for angles close to $90^{\circ}$. Applying the antenna angle correction to the waveform amplitudes increases the maximum amplitude that can be measured from $36.9 \mathrm{mV} / \mathrm{m}$ to $184.5 \mathrm{mV} / \mathrm{m}$.

\section{Foreshock coordinate system}

Magnetic field data from the Cluster FGM experiment (Balogh et al., 1997) at $4 \mathrm{~s}$ resolution and a simple model bow shock were used to determine the position of the foreshock boundary, which is defined by the location where the solar wind magnetic field is tangent to the bow shock. To determine the positions of the Cluster spacecraft within the Earth's foreshock, we used the foreshock coordinate calculation that was first described by Filbert and Kellogg (1979), and later modified by Fitzenreiter et al. (1990) and Cairns et al. (1997). For the complete mathematical description of this coordinate system, please refer to these papers. To simplify calculation of the magnetic field tangent point, we followed the procedure of Cairns et al. (1997) and rotated the positions and magnetic field about the $\mathrm{x}$ GSE axis into a new coordinate system where the magnetic field was completely in the $\mathrm{x}-\mathrm{y}$ plane. Filbert and Kellogg (1979) used a paraboloid in GSE coordinates to represent the bow shock

$x=a_{s}-b_{s}\left(y^{2}+z^{2}\right)$,

where $a_{s}$ is the shock standoff distance from the Earth, and $b_{s}$ determines the perpendicular scale of the shock. We took nominal values of $a_{s}=13.7 R_{E}$ and $b_{s}=0.0223 R_{E}^{-1}$ from Cairns et al. (1997) and scaled $a_{s}$ and $b_{s}$ to move the model bow shock in and out with changes in the solar wind dynamic pressure. Solar wind parameters from the ACE spacecraft, propagated to Cluster's location, were used to determine the appropriate scaling factors. After the point where the solar wind magnetic field is tangent to the model bow shock given by Eq. (1) has been found, we can express the spacecraft locations relative to the foreshock boundary.

Figure 1 illustrates the foreshock coordinate system for Cluster spacecraft 3 on 26 March 2002 at 01:39:58 UT. Before calculating the location of the magnetic tangent point as described above, a rotation about the z GSE axis was applied to the Cluster spacecraft positions and magnetic field data to account for the $\sim 4^{\circ}$ aberration of the magnetosphere symmetry axis due to the Earth's orbital motion through the 


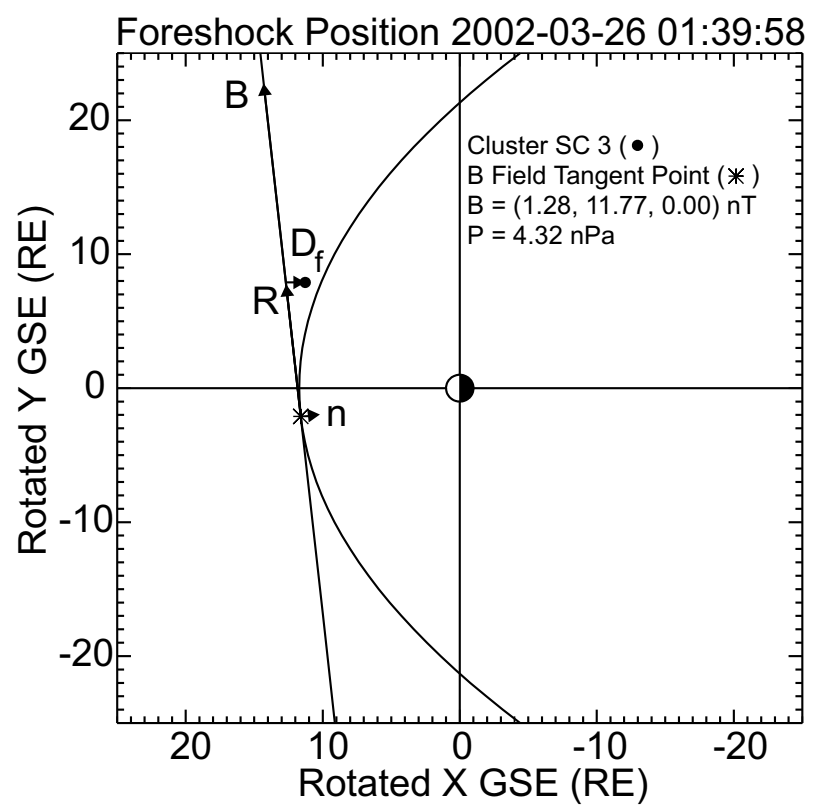

Fig. 1. Illustration of the foreshock coordinate system for Cluster spacecraft 3 on 26 March 2002 at 01:39:58 UT.

interplanetary medium. Solar wind parameters from ACE, propagated to Cluster's location, were used to calculate the actual aberration angle. In Fig. 1, the coordinate $R$ gives the distance from the tangent point to the spacecraft parallel to the solar wind magnetic field. The parameter $D_{f}$ gives the distance from the spacecraft to the tangent field line in the $\mathrm{x}$ GSE direction, or in our case, the distance from the spacecraft to the tangent field line along an axis $\sim 4^{\circ}$ from the $x$ GSE direction when the aberration angle is taken into account. The parameter $D_{f}$ used in this paper and by Cairns et al. (1997) is the same as the parameter DI F F used by Filbert and Kellogg (1979). In this model for the spacecraft location in the foreshock, changes in the parameters $R$ and $D_{f}$ are due to a combination of the spacecraft motion and changes in the direction of the magnetic field, resulting in movement of the tangent point location. When the time-varying solar wind dynamic pressure is used to scale the bow shock model parameters $a_{s}$ and $b_{s}$, changes in the shock location also affect the foreshock coordinates $R$ and $D_{f}$.

\section{Dependence of Langmuir wave amplitudes on fore- shock position}

From 01:30:30 to 01:51:29 UT on 26 March 2002, the Cluster FGM experiment measured a fairly steady magnetic field of about $10 \mathrm{nT}$, dominated by large, northward $B_{z}$ component. When propagation effects are taken into account, ACE upstream magnetic field data show similar behavior. The $x$ GSE component of the solar wind velocity measured by ACE was typically about $-430 \mathrm{~km} / \mathrm{s}$ and the density was near $14 \mathrm{~cm}^{-3}$. The WBD receivers on spacecraft 2,3 , and 4 observed intense waves near the plasma frequency (30-

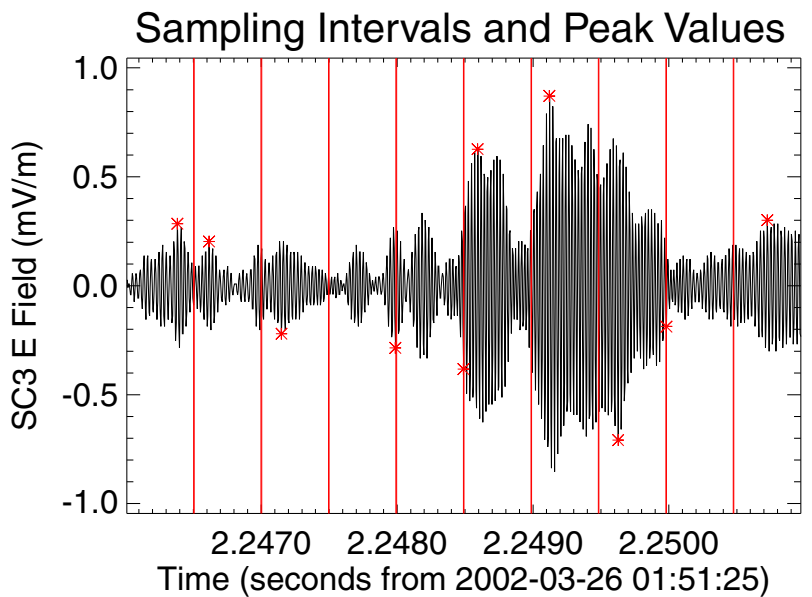

Fig. 2. An example of a typical WBD electric field waveform from $26 \mathrm{March} 2002$. The vertical lines divide the waveform into $10 \mathrm{seg}-$ ments, which were used to determine the peak electric field values for Figs. 3 and 4. The asterisks mark the peak electric field value in each segment. The amplitudes in this figure have not been corrected for the antenna angle to the magnetic field.

$40 \mathrm{kHz}$ ) while Cluster was located in the foreshock from 01:30:30 to 01:51:29 UT. Around 01:51:29 UT, fluctuations in $B_{y}$ with amplitudes greater than $5 \mathrm{nT}$ and fluctuations in $B_{z}$ of $\sim 2-4 \mathrm{nT}$ were observed which caused the foreshock boundary to move earthward of all three spacecraft from approximately 01:51:29-01:51:43 UT and 01:51:5301:52:07 UT. During these intervals, the Cluster spacecraft were located in the solar wind. The final entry into the solar wind during the time period studied in this paper occurred at 01:52:46 UT. The dynamic pressure measured by ACE was steady at $\sim 4.7 \mathrm{nPa}$ between 01:51:29-01:52:46 UT, so the motion of the foreshock boundary during this time period was due to magnetic field fluctuations. Based upon the WBD receiver spectrograms, the spacecraft moved in and out of the foreshock within less than $1 \mathrm{~s}$ of each other during each of the five foreshock boundary crossings from 01:51:29 to 01:52:46 UT. The Cluster spacecraft were separated by only 50 to $100 \mathrm{~km}$, so this implies that the boundary of the foreshock moved over the spacecraft at speeds greater than 50 $100 \mathrm{~km} / \mathrm{s}$.

Figure 2 shows a typical WBD electric field waveform from 26 March 2002 and illustrates how we selected the electric field values for our study. The amplitudes in the Fig. 2 waveform have not been corrected for the angle between the antenna and the magnetic field. As shown by Fig. 2, the Langmuir wave amplitudes can vary considerably on time scales of less than $1 \mathrm{~ms}$. The peak electric field values used to examine the dependence of the amplitudes on position and to construct probability distributions in Sect. 5 were obtained by dividing each waveform into ten $0.5 \mathrm{~ms}$ segments, taking the absolute value of the electric field, and determining the maximum value in each segment. The vertical lines in Fig. 2 show the boundaries of the $0.5 \mathrm{~ms}$ segments and the asterisks mark the peak value in each segment. Segments containing 


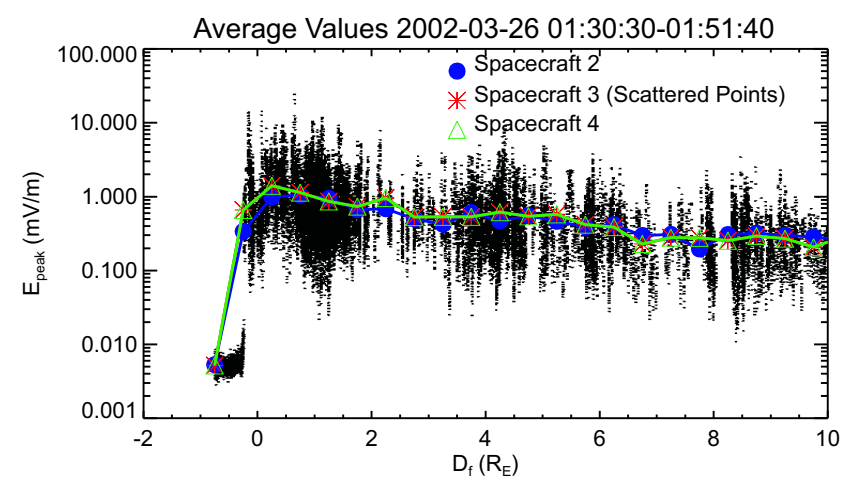

Fig. 3. Dependence of the WBD receiver electric field amplitudes on $D_{f}$ for 26 March 2002 between 01:30:30-01:51:40 UT. The scattered points represent the maximum electric fields in $0.5 \mathrm{~ms}$ windows of each 5-ms waveform snapshot for spacecraft 3 , and the red line represents the average electric field amplitudes in $0.5 R_{E}$ bins of the $D_{f}$ coordinate for spacecraft 3 . The blue and green lines represent the average amplitudes for spacecraft 2 and 4 .

clipped data and segments with maximum amplitudes below our minimum amplitude threshold were discarded because their amplitudes could not be accurately determined.

Figure 3 shows the peak Langmuir wave amplitudes as a function of the foreshock coordinate $D_{f}$ on 26 March 2002 for the time period 01:30:30-01:51:40 UT. The scattered points are the peak electric field amplitudes from spacecraft 3 , determined using the method described in the preceding paragraph. The amplitudes used in Fig. 3 have been corrected for the angle between the WBD antenna and the magnetic field as described in Sect. 2. The red line and points marked with asterisks represent the average values of the electric field for spacecraft 3 in $0.5 R_{E}$ wide bins of $D_{f}$. The green and blue lines represent the average electric field values from spacecraft 2 and 4 , respectively. $D_{f}=0$ marks the model location of the foreshock boundary. The group of points clustered between $D_{f} \sim-1.0$ to $0.0 R_{E}$ and $E \sim 0.003$ to $0.007 \mathrm{mV} / \mathrm{m}$ represent thermal noise (Meyer-Vernet, 1979; Cairns et al., 2000) observed when Cluster was located in the solar wind from 01:51:29-01:51:40 UT. As the thermal noise level does not vary with the orientation of the antenna to the magnetic field, the amplitude correction was not applied to these points.

The sharp rise in the Langmuir wave amplitudes near $D_{f}=-0.2 R_{E}$ indicates the actual position of the foreshock boundary determined by the data. Due to the small separations of the Cluster spacecraft, the dependence of the amplitudes on $D_{f}$ for spacecraft 2, 3 and 4 on 26 March 2002, shown in Fig. 3, are nearly identical. The nearsimultaneous observations of waves with similar amplitudes implies that the foreshock structure is fairly uniform on scales of 50 to $100 \mathrm{~km}$, and supports earlier statistical studies showing that far away from the boundary significant changes in the average wave amplitudes in the foreshock occur on scales greater than $\sim 0.5-1.0 R_{E}$ (Greenstadt et al., 1995; Kasaba et al., 2000).
In Fig. 3, the largest average field values, about $1-2 \mathrm{mV} / \mathrm{m}$ for all three spacecraft, were found in the $0.0<D_{f}<0.5 R_{E}$ bin. The scattered points for spacecraft 3 shown in Fig. 3 indicate that waves with amplitudes from $0.1 \mathrm{mV} / \mathrm{m}$ up to $20 \mathrm{mV} / \mathrm{m}$ were observed in this region. The location of the largest wave amplitudes in Fig. 3 agrees reasonably well with the work of Cairns et al. (1997) which also showed that the largest amplitudes were observed near $D_{f} \sim 0.5 R_{E}$. However, in the data presented by Cairns et al. the wave amplitudes increased slowly with decreasing $D_{f}$ for $1.0<D_{f}<6.0 R_{E}$ but increased by more than a factor of 10 between $0.5<D_{f}<1.0 R_{E}$, creating a distinct high-field region about $1.0 R_{E}$ wide. In the 17 February 2002 case, studied by Sigsbee et al. (2004), the data from the WBD receiver on spacecraft 3 also show a clear boundary between the foreshock and solar wind, and a distinct high-field region of about $1 R_{E}$ wide where the amplitudes increase rapidly. The data shown in Fig. 3 indicate that the Langmuir wave amplitudes increased gradually with decreasing $D_{f}$ throughout the foreshock and that there was not a distinct high-field region near the foreshock boundary for the time period studied on 26 March 2002.

Cairns et al. (1997) estimated that there was $\sim \pm 0.4 R_{E}$ uncertainty in $D_{f}$ due to uncertainties in the values of the $a_{s}$ and $b_{s}$ parameters in the shock model. The offset of the rise in wave amplitudes near the foreshock boundary in Fig. 3 implies that the error in $D_{f}$ is only about $0.2 R_{E}$ for the 01:30:30-01:51:40 UT time period. The fact that no thermal noise points were observed for $D_{f}>0 R_{E}$ also suggests that the bow shock model we used is generally reasonable and gives an accurate calculation of the position in the foreshock when the solar wind magnetic field is steady. The location of the actual foreshock boundary in Fig. 3 is quite clear and is the same for all three spacecraft, which implies that the positions of the spacecraft relative to the foreshock boundary are well-defined for the 01:30:30-01:51:40 UT time period, even if there is a small offset in the absolute location of the boundary determined by the electric field amplitudes. However, the error in $D_{f}$ was considerably larger when the foreshock boundary was in motion from 01:51:43-01:52:46 UT. More than $85 \%$ of the values greater than $0.01 \mathrm{mV} / \mathrm{m}$ measured by spacecraft 3 between 01:51:43 and 01:52:46 UT were placed at $D_{f}<0 R_{E}$, even though these waves were observed in the foreshock. Simulations have shown that when the solar wind magnetic field is turbulent, it is possible for a spacecraft to be magnetically connected to the bow shock, even when it is upstream of the nominal foreshock boundary and that the spread in the boundary location can be as much as $1 R_{E}$ (Zimbardo and Veltri, 1996). This is a likely explanation why the position calculation was not accurate for 01:51:43 to $01: 52: 46$ UT.

Figure 3 shows that for $2.0<D_{f}<10.0 R_{E}$, the average corrected amplitude of the waves observed in the foreshock by the WBD receiver on 26 March 2002 ranged from $0.3 \mathrm{mV} / \mathrm{m}$ to $0.5 \mathrm{mV} / \mathrm{m}$. In the 17 February 2002 data studied by Sigsbee et al. (2004), the average corrected amplitude for this range of $D_{f}$ values was usually between $0.7 \mathrm{mV} / \mathrm{m}$ 
to $0.8 \mathrm{mV} / \mathrm{m}$, with amplitudes varying from $0.1 \mathrm{mV} / \mathrm{m}$ up to $4.0 \mathrm{mV} / \mathrm{m}$. The largest amplitude waves observed on 17 February 2002 extended to the maximum range of the WBD receiver $(36.9 \mathrm{mV} / \mathrm{m})$ and had corrected peak amplitudes up to $100 \mathrm{mV} / \mathrm{m}$ near the foreshock boundary. However, the largest corrected amplitudes for 26 March 2002, shown in Fig. 3, were only $20.0 \mathrm{mV} / \mathrm{m}$. Waves with corrected amplitudes up to $60 \mathrm{mV} / \mathrm{m}$ were observed from 01:51:43 to 01:52:46 UT when the foreshock boundary was in rapid motion due to fluctuations of the magnetic field, but were not included in Fig. 3 as the error in $D_{f}$ was too large due to the boundary motions. On 17 February 2002, the background magnetic field was more closely aligned with the WBD antennas than it was on 26 March 2002. The less favorable orientation of the spacecraft with respect to the magnetic field on 26 March 2002 is the most likely reason why the amplitudes of the waves observed on this day were smaller than those observed on 17 February 2002. However, other factors may have also contributed to the difference in the wave amplitudes. For the time period of interest on 26 March 2002, the average Alfvén Mach number calculated using ACE data was 6.6. For the time period studied on 17 February 2002, the average Alfvén Mach number was 5.7. In a study of electrostatic waves near the Earth's bow shock using AMPTE IRM data, Onsager et al. (1989) found that the wave amplitudes decreased with increasing Alfvén Mach number. Our observations appear to be consistent with the results of this study.

\section{Amplitude probability distributions}

The statistics of the electric field waveform amplitudes in the foreshock can also be used to examine possible growth mechanisms for the Langmuir waves, such as stochastic growth theory. Stochastic growth theory considers the behavior of waves subject to a randomly varying growth rate (Robinson, 1995). Spatial inhomogeneities and time-varying perturbations in the plasma cause the appearance of regions of positive and negative growth rate. Waves propagating through these regions grow at a rate that fluctuates randomly around the mean. According to Cairns and Robinson (1999, and references therein), the amplitude probability distribution is Gaussian in $\log E$

$P(\log E)=(\sigma \sqrt{2 \pi})^{-1} e^{-(\log E-\mu)^{2} / 2 \sigma^{2}}$,

where $\mu$ and $\sigma$ are the average and standard deviation of $\log E$. Using Wind TDS data, Bale et al. (1997) found that the amplitude probability distribution for Langmuir waves in the foreshock actually resembled a power law $P(\log E) \propto E^{-0.99}$. Cairns and Robinson (1997) found that the observed ISEE 1 electric field amplitude probability distribution agreed with the stochastic growth theory prediction given by Eq. (2) for a constant spacecraft location relative to the foreshock boundary. For periods with large variation in the spacecraft location, they found a power law
$P(\log E) \propto E^{-0.8 \pm 0.3}$. This led Cairns and Robinson to conclude that the power law distribution of amplitudes was the result of the convolution of the actual amplitude probability distribution with the spacecraft motion.

To construct the probability distributions for the WBD receiver data, we divided the full $123 \mathrm{~dB}$ amplitude range into $2.5 \mathrm{~dB}$ wide bins. Since the gain setting of the WBD Plasma Wave Receiver increases or decreases in $5 \mathrm{~dB}$ steps, choosing an amplitude bin width of $2.5 \mathrm{~dB}$ will allow the bins in the different gain states to exactly match in the overlapping portions of their amplitude ranges. We found the peak amplitudes, as described in Sect. 4, and then determined the percentage of waveforms in each amplitude bin. We first constructed amplitude probability distributions including electric fields measured for all values of $D_{f}$. To account for the fact that Cluster did not spend equal amounts of time at each position, we weighted every count in the electric field amplitude bins by the number of points in the $D_{f}$ bin with the fewest points divided by the number of points in the $D_{f}$ bin where the measurement was taken. To investigate the possibility that the behavior of the waves may vary with position in the foreshock, we also constructed probability distributions using only measurements taken in $1 R_{E}$ bins of $D_{f}$ for the range $-1.0<D_{f}<10.0 R_{E}$. No weighting factors were applied to the counts used to construct the probability distributions in the $1 R_{E}$ bins of $D_{f}$.

Figure $4 \mathrm{a}, \mathrm{b}$ and $\mathrm{c}$ show the probability distributions from 26 March 2002 for the time period 01:30:30-01:51:40 UT, using weighted counts for all values of $D_{f}$ less than $10 R_{E}$ for spacecraft 2, 3 and 4, respectively. The dotted lines show the fits to the Gaussian function predicted by stochastic growth theory. The probability distributions are similar for all three spacecraft, as would be expected based upon the agreement of average waveform amplitudes for all three spacecraft shown in Fig. 3. The probability distributions for all values of $D_{f}$ from the three spacecraft fit reasonably well to a Gaussian over the entire amplitude range shown in Fig. 4. In contrast, the probability distribution for all values of $D_{f}$ from Cluster spacecraft 3 on 17 February 2002, discussed in Sigsbee et al. (2004), appeared to be Gaussian for amplitudes less than $1-2 \mathrm{mV} / \mathrm{m}$ but resembled a power law for larger amplitudes.

Figure $4 \mathrm{~d}$ shows the probability distributions from selected $1 R_{E}$ bins in $D_{f}$ for spacecraft 4 for the time period 01:30:30-01:51:40 UT on 26 March 2002 with fits to a Gaussian (dotted line). The probability distributions for the $0.0<D_{f}<1.0 R_{E}, \quad 1.0<D_{f}<2.0 R_{E}, 2.0<D_{f}<3.0 R_{E}$, and $3.0<D_{f}<4.0 R_{E}$ bins fit well to the prediction of stochastic growth theory at all amplitudes, with reduced chi-squared values of 4.0, 6.8, 2.4, and 1.1, respectively. The probability distributions for the $1 R_{E}$ bins of $D_{f}$ fit better to a Gaussian than the probability distributions including all values of $D_{f}$, which had reduced chi-squared values of 12.2 for spacecraft 2, 9.1 for spacecraft 3 , and 8.6 for spacecraft 4 . The probability distributions calculated in bins of $D_{f}$ for the 17 February 2002 case studied by Sigsbee et al. (2004) also appeared to be Gaussian at all distances to the foreshock boundary. When 

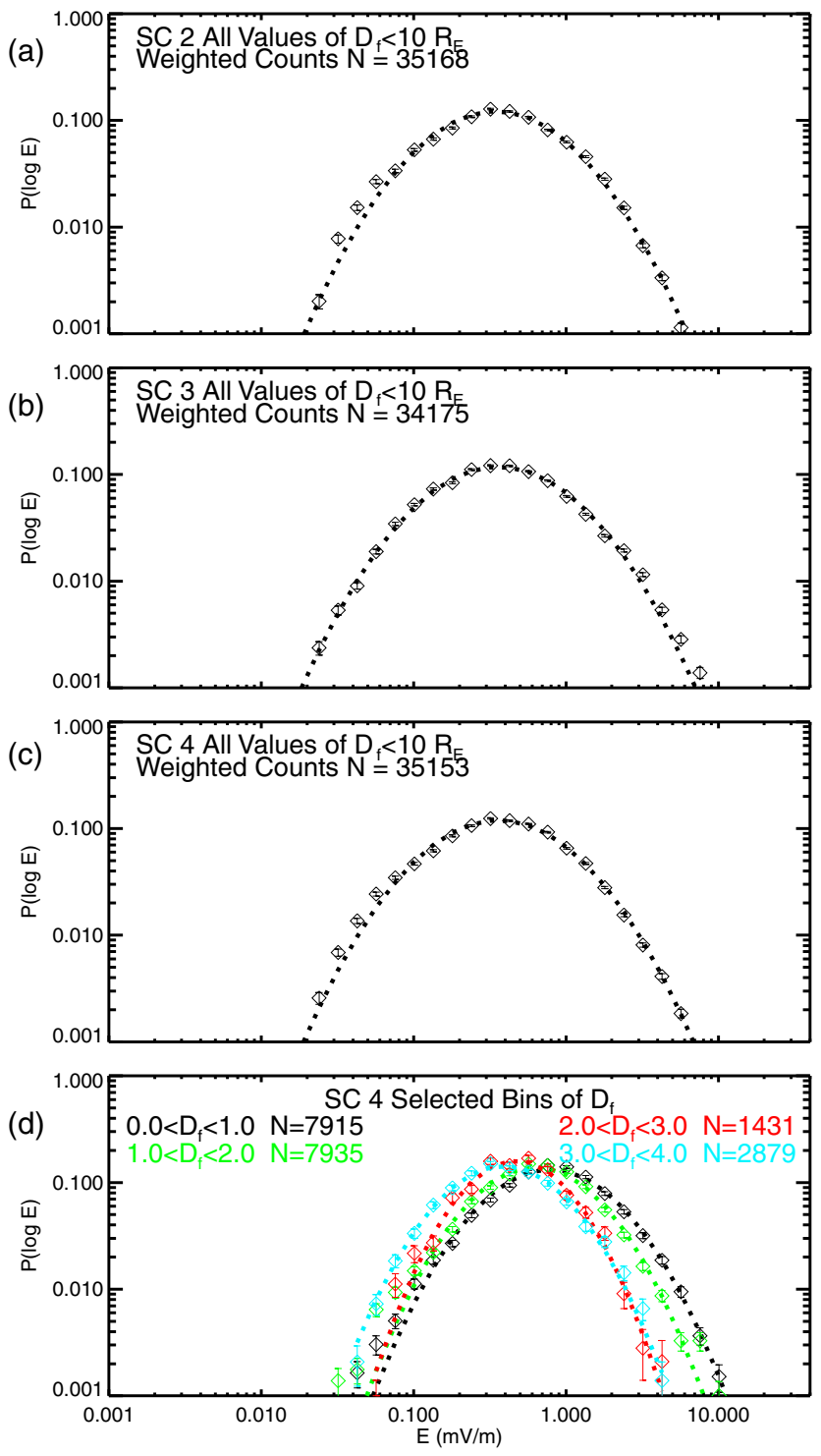

Fig. 4. Probability distributions for the electric field waveform amplitudes observed by the Cluster WBD Plasma Wave Receiver on 26 March 2002. The probability distributions for all values of $D_{f}$ using weighted counts for (a) spacecraft 2, (b) spacecraft 3, and (c) spacecraft 4 . The dashed lines show the fit to the Gaussian function predicted by stochastic growth theory. (d) Probability distributions from spacecraft 4 for selected $1 R_{E}$ bins in $D_{f}$.

the data were organized into bins of $D_{f}$, the probability distributions for 26 March 2002 and for the 17 February 2002 case studied by Sigsbee et al. both show that the center of the probability distributions shifts gradually toward lower amplitudes as the spacecraft goes deeper into the foreshock, in a manner consistent with the average amplitudes for each bin. This is also consistent with the results of Filbert and Kellogg (1979) and Cairns and Robinson (1999).

In the 17 February 2002 data, it appeared that the power law tail on the probability distribution calculated using weighted counts from all values of $D_{f}$ was effectively the result of summing the distributions calculated in different bins of position over $D_{f}$. On 17 February 2002, the average corrected wave amplitudes increased by less than $0.1 \mathrm{mV} / \mathrm{m}$ per $R_{E}$ with decreasing $D_{f}$ deep inside the foreshock, but they increased by more than $5 \mathrm{mV} / \mathrm{m}$ per $R_{E}$ within $1.5 R_{E}$ of the foreshock boundary. Close to the foreshock boundary, corrected amplitudes reached values near $100 \mathrm{mV} / \mathrm{m}$ on 17 February 2002. Although the 17 February 2002 probability distributions for each bin of $D_{f}$ values were Gaussian in form, the sudden increase in the center electric field amplitude of the distributions close to the foreshock boundary caused their sum to have a power law tail at large amplitudes. The 17 February 2002 data clearly support the results of Boshuizen et al. (2001), which showed that spatially averaged probability distributions including measurements from a wide range of $D_{f}$ values will appear to be power laws.

The reason why the 26 March 2002 probability distributions for all values of $D_{f}$ shown in Fig. 4 do not have a power law tail at large amplitudes can be understood by examining Fig. 3. The average corrected amplitudes in Fig. 3 increase steadily by about $0.1-0.2 \mathrm{mV} / \mathrm{m}$ per $R_{E}$ right up to the foreshock boundary. Since the shift of the centers of the probability distributions for individual bins of $D_{f}$ for 26 March 2002 was more constant throughout the foreshock, the total distribution for all values of $D_{f}$ still appears to be Gaussian.

\section{Effects of non-optimal auto-ranging on statistics}

Nearly every study of waves in the foreshock has relied upon data from an instrument that either has used some sort of automatic ranging or automatic selection of waveform captures based upon amplitude. However, automatic ranging can introduce bias into the amplitude statistics. Figure 5 shows the percentages of waveforms in each gain state rejected due to clipping or because the peak amplitudes were below the minimum amplitude threshold for spacecraft 3 for both 26 March 2002 and 17 February 2002. On 17 February 2002, a total of $18.2 \%$ of the waveforms were rejected. On 26 March 2002, $21.2 \%$ of the waveforms were rejected.

Figure 5a shows that on 17 February 2002 less than $40 \%$ of the waveforms in the $15 \mathrm{~dB}$ to $50 \mathrm{~dB}$ gain states were rejected, and the $60 \mathrm{~dB}$ gain state was the only setting where more than $50 \%$ of the waveforms were rejected. Figure $5 \mathrm{~b}$ shows that on 26 March 2002, less than $40 \%$ of the waveforms were rejected in the $30 \mathrm{~dB}$ to $55 \mathrm{~dB}$ gain states, which is a considerably smaller portion of the amplitude range than on 17 February 2002. Also, Fig. 5b shows on 26 March 2002 that $50 \%$ or more of the waveforms were rejected in the $0 \mathrm{~dB}$ to $20 \mathrm{~dB}$ gain states and in the $65 \mathrm{~dB}$ to $75 \mathrm{~dB}$ gain states. Although the average wave amplitudes observed on 26 March 2002 appear to have been distinctly lower than on 17 February 2002, elimination of waveforms due to non-optimal autoranging may help explain why there were so few waves observed with uncorrected amplitudes greater than $10 \mathrm{mV} / \mathrm{m}$ on 26 March 2002. In the 26 March 2002 data, 4.6\% of the waveforms were rejected due to clipping, but only $4.3 \%$ were rejected due to clipping in the 17 February 2002 data. The 
largest amplitude waves observed on 26 March 2002 were very bursty and transient in nature, and the $0.1 \mathrm{~s}$ gain update rate appeared to be much slower than the time scales on which the amplitudes of the waves changed. This probably resulted in the WBD receivers not always being set in the most appropriate gain state, as the automatic gain control attempted to compensate for the rapidly changing amplitudes, and can also explain why a larger percentage of waveforms were rejected on 26 March 2002 because they fell below the minimum amplitude threshold.

The effects of the automatic gain control may offer an explanation why the probability distributions sometimes vary from the expected shape. Manually setting the gain state is one way to deal with this problem, however, the probability distribution obtained will only be valid for a limited amplitude range. On 17 February 2002, the gain on spacecraft 4 was manually set to $0 \mathrm{~dB}$. Only $0.03 \%$ of the waveforms observed by spacecraft 4 were excluded due to clipping, but more than $95 \%$ of the waveforms had to be rejected because they fell below the minimum amplitude threshold. The result was that the probability distributions constructed for spacecraft 4 on 17 February 2002 by Sigsbee et al. (2004) were only valid for uncorrected amplitudes between $2.0 \mathrm{mV} / \mathrm{m}$ and $36.9 \mathrm{mV} / \mathrm{m}$. The WBD receiver was designed with a great deal of overlap between the amplitude ranges for the various gain settings, so it may be possible to correct for the effects of non-optimal auto-ranging by comparing the results from different gain states for the same amplitude range. Studies are currently underway to do this comparison.

\section{Conclusions}

The Cluster WBD Plasma Wave Receiver observations on 26 March 2002 are consistent with earlier studies of Langmuir waves in the foreshock conducted using data from Wind and ISEE 1 (Bale et al., 1997; Cairns and Robinson, 1997). Typical Langmuir wave electric field amplitudes in the Earth's foreshock are on the order of a few $\mathrm{mV} / \mathrm{m}$ or less, but waves with amplitudes greater than $10 \mathrm{mV} / \mathrm{m}$ are occasionally observed. The largest amplitude waves were observed near the boundary of the foreshock, consistent with earlier studies. However, the waves observed on 26 March 2002 were generally lower in amplitude than the waves on 17 February 2002, discussed by Sigsbee et al. (2004), and the high-field region near the foreshock boundary was less distinct than on 17 February 2002. The behavior of the magnetic field and the higher Alfvén Mach number on 26 March 2002 may explain these differences. The amplitude of the Langmuir waves fell off at a fairly steady rate with increasing distance from the foreshock boundary on 26 March 2002. This behavior is different from the results of Sigsbee et al. (2004) and Cairns et al. (1997), which showed a sudden decrease close to the boundary, and a more gradual decrease far from the boundary. The dependence of the amplitudes on distance to the foreshock boundary was the same for Cluster spacecraft 2, 3, and 4 during the time period studied on 26 March 2002.
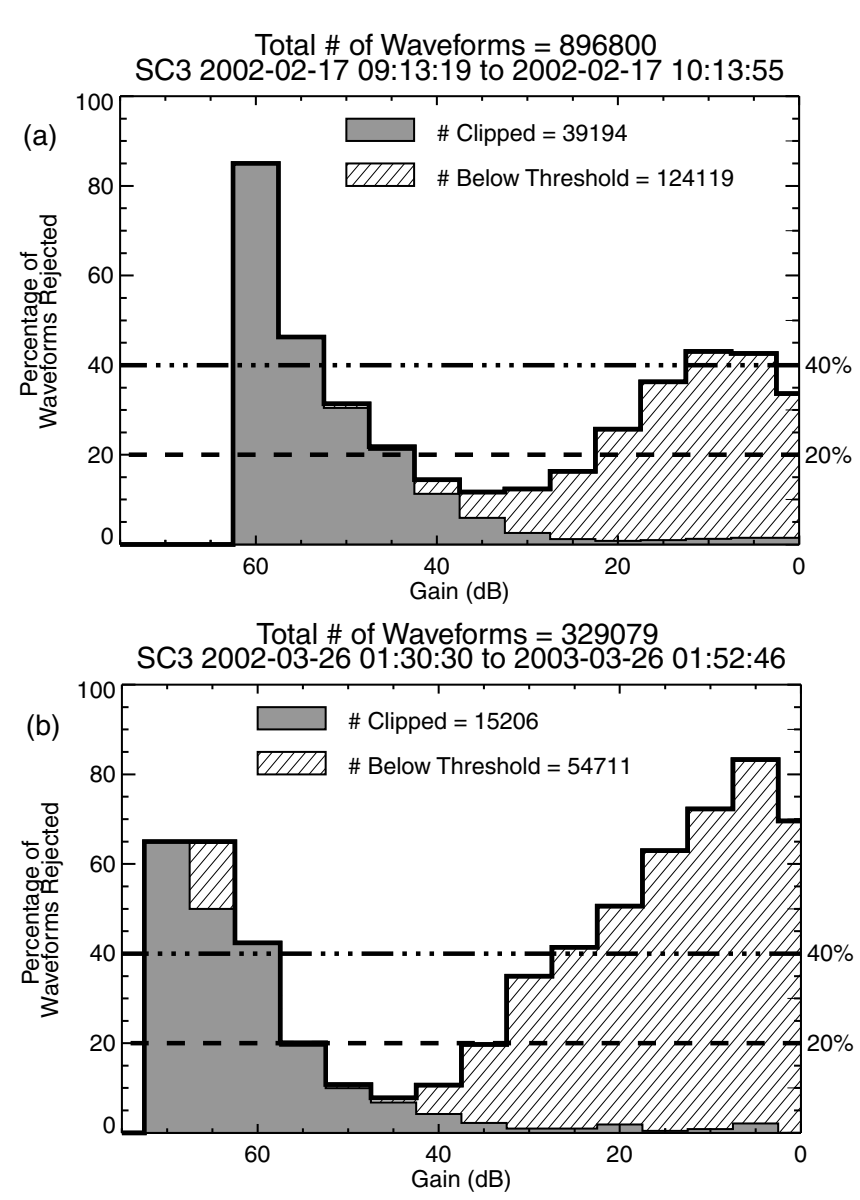

Fig. 5. Histograms of the percentages of waveforms in each gain state rejected for (a) spacecraft 3 on 17 February 2002 and (b) spacecraft 3 on 26 March 2002. The solid black line indicates the total percentages of waveforms rejected in each gain state. The gray shaded area indicates the percentage of clipped waveforms and the line-filled area indicates the percentage of waveforms below the minimum amplitude threshold.

Comparison with the predictions of stochastic growth theory shows that the Langmuir wave observations for 26 March 2002 followed the log-normal statistics predicted by stochastic growth theory. Power law deviations from the stochastic growth theory prediction at large amplitudes have been well-documented (Bale et al., 1997; Cairns and Robinson, 1999; Sigsbee et al., 2004). These deviations generally occur when electric field amplitudes from a large range of distances from the foreshock boundary are included in the probability distribution. However, even when data from a large range of distances to the foreshock boundary were included in the 26 March 2002 probability distributions, they followed lognormal statistics. The behavior of the waves on 26 March 2002 may have been physically different from what has been observed in previous studies, but the errors in the $D_{f}$ calculation close to the foreshock boundary and the effects of non-optimal auto-ranging of the instrument could also have influenced the statistics. 
Acknowledgements. We thank the ACE SWEPAM and MAG Instrument Teams and the ACE Science Data Center for providing the ACE data. This work was supported by NASA/GSFC grant NAG59974.

Topical Editor T. Pulkkinen thanks S. D. Bale and another referee for their help in evaluating this paper.

\section{References}

Bale, S. D., Burgess, D., Kellogg, P. J., Goetz, K., and Monson, S. J.: On the amplitude of intense Langmuir waves in the terrestrial electron foreshock, J. Geophys. Res., 102, 11 281-11 286, 1997.

Bale, S. D., Larson, D. E., Lin, R. P., Kellogg, P. J., Goetz, K., and Monson, S. J.: On the beam speed and wavenumber of intense electron plasma waves near the foreshock edge, J. Geophys. Res., 105, 27 353-27 367, 2000.

Balogh, A., Dunlop, M. W., Cowley, S. W. H., Southwood, D. J., Thomlinson, J. G., Glassmeier, K. H., Musmann, G., Luhr, H., Buchert, S., Acuna, M. H., Fairfield, D. H., Slavin, J. A., Riedler, W., Schwingenschuh, K., and Kivelson, M. G.: The Cluster magnetic field investigation, Space Sci. Rev., 79, 65-91, 1997.

Boshuizen, C. R., Cairns, I. H., and Robinson, P. A.: Stochastic growth theory of spatially-averaged distributions of Langmuir fields in Earth's foreshock, Geophys. Res. Lett., 28, 3569-3572, 2001.

Cairns, I. H. and Robinson, P. A.: First test of stochastic growth theory for Langmuir waves in Earth's foreshock, Geophys. Res. Lett., 24, 369-372, 1997.

Cairns, I. H. and Robinson, P. A.: Strong evidence for stochastic growth of Langmuir-like waves in Earth's foreshock, Phys. Rev. Lett., 82, 3066-3069, 1999.

Cairns, I. H., Robinson, P. A., Anderson, R. R., and Strangeway, R. J.: Foreshock Langmuir waves for unusually constant solar wind conditions: Data and implications for foreshock structure, J. Geophys. Res., 102, 24 249-24 264, 1997.

Cairns, I. H., Robinson, P. A., and Anderson, R. R.: Thermal and driven stochastic growth of Langmuir waves in the solar wind and Earth's foreshock, Geophys. Res. Lett., 27, 61-64, 2000.

Etcheto, J. and Faucheux, M.: Detailed study of electron plasma waves upstream of the Earth's bow shock, J. Geophys. Res., 89, 6631-6653, 1984.

Filbert, P. C. and Kellogg, P. J.: Electrostatic noise at the plasma frequency beyond the Earth's bow shock, J. Geophys. Res., 84, 1369-1381, 1979.

Fitzenreiter, R. J., Klimas, A. J., and Scudder, J. D.: Detection of bump-on-tail reduced electron velocity distributions at the electron foreshock boundary, Geophys. Res. Lett., 11, 496-499, 1984.
Fitzenreiter, R. J., Scudder, J. D., and Klimas, A. J.: Threedimensional analytical model for the spatial variation of the foreshock electron distribution function: Systematics and Comparisons with ISEE observations, J. Geophys. Res., 95, 4155-4173, 1990.

Greenstadt, E. W., Crawford, G. K., Strangeway, R. J., Moses, S. L., and Coroniti, F. V.: Spatial distribution of electron plasma oscillations in the Earth's foreshock at ISEE 3, J. Geophys. Res., 100, 19933-19939, 1995.

Gurnett, D. A., Huff, R. L., and Kirchner, D. L.: The Wide-Band Plasma Wave Investigation, Space Sci. Rev., 79, 195-208, 1997.

Kasaba, Y., Matsumoto, H., Omura, Y., Anderson, R. R., Mukai, T., Saito, Y., Yamamoto, T., and Kokubun, S.: Statistical studies of plasma waves and backstreaming electrons in the terrestrial electron foreshock observed by Geotail, J. Geophys. Res., 105, 79-103, 2000.

Meyer-Vernet, N.: On natural noises detected by antennas in plasmas, J. Geophys. Res., 84, 5373-5377, 1979.

Onsager, T. G., Holzworth, R. H., Koons, H. C., Bauer, O. H., Gurnett, D. A., Anderson, R. R., Lühr, H., and Carlson, C. W.: Highfrequency electrostatic waves near Earth's bow shock, J. Geophys. Res., 94, 13 397-13 408, 1989.

Robinson, P. A.: Stochastic wave growth, Phys. Plasmas, 2, 14661479, 1995.

Robinson, P. A. and Cairns, I. H.: Maximum Langmuir fields in planetary foreshocks determined from the electrostatic decay threshold, Geophys. Res. Lett., 22, 2657-2660, 1995.

Robinson, P. A., Cairns, I. H., and Gurnett, D. A.: Clumpy Langmuir waves in type III radio sources: comparison of stochasticgrowth theory with observations, Astrophys. J., 407, 790-800, 1993.

Sigsbee, K., Kletzing, C. A., Gurnett, D. A., Pickett, J. S., Balogh, A., and Lucek, E.: The dependence of Langmuir wave amplitudes on position in Earth's foreshock, Geophys. Res. Lett., 31, L07805, doi:10.1029/2004GL019413, 2004.

Woolliscroft, L. J. C., Alleyne, H., Dunford, C. M., Sumner, A., Thompson, J. A., Walker, S. N., Yearby, K. H., Buckley, A., Chapman, S., and Gough, M. P.: The Digital Wave Processing Experiment on Cluster, Space Sci. Rev., 79, 209-231, 1997.

Zakharov, V. E.: Collapse of Langmuir waves, Sov. Phys. JETP, 35, 908-914, 1972.

Zimbardo, G. and Veltri, P.: Spreading and intermittent structure of the upstream boundary of planetary magnetic foreshocks, Geophys. Res. Lett., 23, 793-796, 1996. 